Journal of Knot Theory and Its Ramifications

Vol. 16, No. 10 (2007) 1469-1472

(C) World Scientific Publishing Company

\title{
AUTHOR INDEX \\ Volume 16
}

Andersen, J. E. \& Ueno, K., Geometric construction of modular functors from conformal field theory

Apostolakis, N. \& Kaiser, U.,

Kauffman state sums and bracket deformation

Bar-Natan, D.,

Fast Khovanov homology computations

Barnes, D. W.,

On Sikora's spectral sequences

Bellingeri, P. \& Godelle, E., Positive presentations of surface braid groups

Blain, P., Bowlin, G., Fleming, T., Foisy, J., Hendricks, J. \& Lacombe, J., Some results on intrinsically knotted graphs

Bowlin, G., See Blain

Buck, D. \& Marcotte, C. V., Classification of tangle solutions for integrases, a protein family that changes DNA topology

Burton, B. A.,

Structures of small closed non-orientable 3-manifold triangulations

Clay, A. \& Rolfsen, D., Densely ordered braid subgroups

Collins, P., Universal trellises

Dabkowska, M. A., Dabkowski, M. K., Harizanov, V. S., Przytycki, J. H. \& Veve, M. A.,

Compactness of the space of left orders
2(2007) 127

7 (2007) 809

3 (2007) 243

7 (2007) 833

9 (2007) 1219

6 (2007) 749

6 (2007) 749

$8(2007) 969$

5 (2007) 545

7 (2007) 869

$4(2007) 471$

3 (2007) 257
Dabkowski, M. K. \& Sahi, R. K.,

New invariant of 4-moves

Dabkowski, M. K., See Dabkowska

Da̧bkowski, M. K., Ishiwata, M. \& Przytycki, J.H.,

5-Move equivalence classes of links and theiralgebraic invariants

Dar, A.,

Amphicheiral knots with every even crossing number

De Wit, D. \& Links, J., Where the LinksGould invariant first fails to distinguish nonmutant prime knots

De Wit, D.,

The 2-bridge knots of up to 16 crossings

Eisermann, M. \& Lamm, C., Equivalence of symmetric union diagrams

Fauser, B. \& Jarvis, P. D.,

The Dirichlet Hopf algebra of arithmetics

Ferrand, E.,

Arrangements of nested curves

Fleming, T. \& Pommersheim, J.,

Intrinsicallylinked graphs and the Sato-Levine invariant

Fleming, T., See Blain

Foisy, J., See Blain

Futer, D., Involutions of knots that fix unknotting tunnels
$10(2007) 1261$

3 (2007) 257

10 (2007) 1413

$1(2007) 43$

8 (2007) 1021

8 (2007) 997

7 (2007) 879

4 (2007) 379

2 (2007) 217

6 (2007) 761

6 (2007) 749

6 (2007) 749

6 (2007) 741 
Gilmer, P. M. \& Harris, J. M.,

On the Kauffman bracket skein module of the quaternionic manifold

Gilmer, P. M.,

Remarks on congruence of 3-manifolds

Godelle, E., See Bellingeri

Grishanov, S. A., Meshkov, V. R. \& Omel'chenko, A. V.,

Kauffman-type polynomial invariants for doubly periodic structures

Harizanov, V. S., See Dabkowska

Harrell, N. \& Nelson, S., Quandles and linking number

Harris, J. M., See Gilmer

Helme-Guizon, L., The coefficients of the HOMFLYPT and the Kauffman polynomials are pointwise limits of vassiliev invariants

Hendricks, J., See Blain

Hinojosa, G., A wild knot $\mathbb{S}^{2} \hookrightarrow$ $\mathbb{S}^{4}$ as limit set of a Kleinian group: Indra's pearls in four dimensions

Horiuchi, S.,

The Jacobi diagram for a $C_{n}$-move and the HOMFLY polynomial

Hrencecin, D. \& Kauffman, L. H.,

Biquandles for virtual knots

Hsieh, C.-C.,

Combinatoric and diagrammatic study in knot theory

Ishiwata, M., See Dąbkowski

Jablan, S. \& Sazdanović, R., Unlinking number and unlinking gap
1 (2007) 103

$10(2007) 1357$

9 (2007) 1219

$6(2007) 779$

3 (2007) 257

$10(2007) 1283$

1 (2007) 103

2 (2007) 203

6 (2007) 749

8 (2007) 1083

$2(2007) 227$

10 (2007) 1361

9 (2007) 1235

10 (2007) 1413

10 (2007) 1331
Jackson, D. M., Moffatt, I. \& Morales, A.,

On the group-like behavior of the Le-MurakamiOhtsuki invariant

Jang, H. J., Lee, S. Y. \& Seo, M.,

Casson knot invariants of periodic knots with rational quotients

Jarvis, P. D.,

See Fauser

Jordan-Santana, M., A geometric proof that the singular braid group is torsion free

Kaiser, U., See Apostolakis

Kalfagianni, E. \& Lin, X.-S., Seifert surfaces, commutators and Vassiliev invariants

Kanenobu, T. \& Tsuji, K., Groups of two-braid virtual knots

Kang, E., Seifert surfaces in knot complements

Kauffman, L. H. \& Lomonaco Jr, S. J., $q$-Deformed spin networks, knot polynomials and anyonic topological quantum computation

Kauffman, L. H., Pendulum traced curves and damped oscillations in the plane

Kauffman, L. H., See Hrencecin

Kauffman, L. H., State models for knot polynomials - An introduction

Ko, K. H. \& Song, W. T., Seifert matrices of periodic knots

Kurlin, V. \& Lines, D., Peripherallyspecified homomorphs of link groups

Kurlin, V.,

Three-page encoding and complexity theory for spatial graphs
6 (2007) 699

4 (2007) 379

8 (2007) 1067

7 (2007) 809

$10(2007) 1295$

6 (2007) 671

8 (2007) 1053

3 (2007) 267

10 (2007) 1451

$10(2007) 1361$

7 (2007) 899

1 (2007) 45

6 (2007) 719

1 (2007) 59 
Lacombe, J. See Blain

Lambropoulou, S., $L$-Moves and Markov theorems

Lamm, C., See Eisermann

Lauda, A. D. \& Pfeiffer, H., State sum construction of two-dimensional openclosed topological quantum field theories

Lee, S. Y., See Jang

Levine, J., Concordance of boundary links

Lin, X.-S., See Kalfagianni

Lines, D. See Kurlin

Links, J., See De Wit

Lins, S., Combinatorial DehnLickorishtwists and framed link presentations of 3-manifolds revisited

Lomonaco Jr., S. J., See Kauffman

Mackaay, M., Turner, P. \& Vaz, P.,

A remark on Rasmussen's invariant of knots

Manturov, V. O., Khovanov homology for virtual knots with arbitrary coefficients

Marcotte, C. V., See Buck

Martins, J. F., Categorical groups, knots and knotted surfaces

Mattman, T. W. \& Sizemore, O.,

Bounds on the crosscap number of torus knots

Mcatee Ganatra, J. M., Knots of constant curvature

Meshkov, V. R., See Grishanov

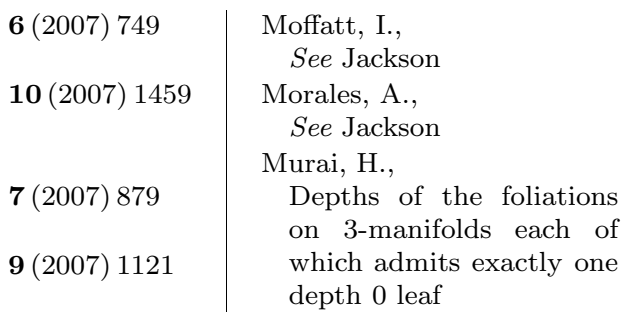

Moffatt, I., See Jackson

Morales, A., See Jackson

Murai, H., Depths of the foliations on 3-manifolds each of which admits exactly one depth 0 leaf

Murillo, G., Nelson, S. \& Thompson, A.,

Matrices and finite Alexander quandles

Nelson, S., See Harrell

Nelson, S. See Murillo

Niebrzydowski, M., Biquandle longitude invariant of long virtual knots

O'Connor, A., Podlesny, B., Soriano, N., Trapp, R. \& Wall, D.,

Clasp moves and stick number

Ogasa, E.,

Ribbon-moves of 2-knots: The torsion linking pairing and the $\widetilde{\eta}$-invariants of 2-knots

Omel'chenko, A. V., See Grishanov

Pfeiffer, H., See Lauda

Podlesny, B. See O'Connor

Pommersheim, J., See Fleming

Przytycki, J. H., See Dabkowska

Przytycki, J. H., See Dąbkowski

Radford, D. E., On the tensor product of an oriented quantum algebra with itself

Rolfsen, D., See Clay

6 (2007) 699

6 (2007) 699

5 (2007) 641

6 (2007) 769

$10(2007) 1283$

6 (2007) 769

10 (2007) 1393

9 (2007) 1165

5 (2007) 523

6 (2007) 779

9 (2007) 1121

9 (2007) 1165

6 (2007) 761

3 (2007) 257

10 (2007) 1413

7 (2007) 929

7 (2007) 869

6 (2007) 789

Rubinsztein, R. L., Topological quandles and invariants of links

Sahi, R. K.,

10 (2007) 1261 See Dabkowski 
Satoh, S., A note on the shadow cocycle invariant of a knot with a base point

Sazdanović, R., See Jablan

Seo, M., See Jang

Shumakovitch, A. N., Rasmussen invariant, slice-Bennequin inequality, and sliceness of knots

Sizemore, O., See Mattman

Song, W. T., See Ko

Soriano, N., See O'Connor

Thompson, A., See Murillo

Tourtchine, V., On the other side of the bialgebra of Chord diagrams

Trapp, R., See O'Connor

Tsuji, K., See Kanenobu

$\mathbf{7}(2007) 959$
$\mathbf{1 0}(2007) 1331$
$\mathbf{4}(2007) 439$
$\mathbf{1 0}(2007) 1403$
$\mathbf{8}(2007) 1043$
$\mathbf{1}(2007) 45$
$\mathbf{9}(2007) 1165$
$\mathbf{6}(2007) 769$
$\mathbf{5}(2007) 575$
$\mathbf{9}(2007) 1165$
$\mathbf{6}(2007) 671$

Tsukamoto, T. \& Yasuhara, A.,

A factorization of the Conway polynomial and covering linkage invariants

Turner, P., See Mackaay

Ueno, K., See Andersen

Vaz, P., See Mackaay

Veve, M. A., See Dabkowska

Viro, J., Linking number in a projective space as the degree of a map

Volić, I., A survey of Bott-Taubes integration

Wall, D., See O'Connor

Yasuhara, A., See Tsukamoto

Yun, K.-H., Multi-variable Alexander polynomial of a plat
5 (2007) 631

3 (2007) 333

2 (2007) 127

3 (2007) 333

3 (2007) 257

4 (2007) 489

$1(2007) 1$

9 (2007) 1165

5 (2007) 631

4 (2007) 499 